\title{
Healthcare Automation System by Using Cloud-Based Telemonitoring Technique for Cardiovascular Disease Classification
}

\author{
Basudev Halder, Neotia Institute of Technology, Management, and Science, Kolkata, India \\ Sucharita Mitra, Netaji Nagar Day College, Kolkata, India \\ Madhuchhanda Mitra, University of Calcutta, Kolkata, India
}

\begin{abstract}
This paper illustrates the cloud-based telemonitoring framework that implements healthcare automation system for myocardial infarction (MI) disease classification. For this purpose, the pathological feature of ECG signal such as elevated ST segment, inverted T wave, and pathological Q wave are extracted, and MI disease is detected by the rule-based rough set classifier. The information system involves pathological feature as an attribute and decision class. The degree of attributes dependency finds a smaller set of attributes and predicted the comprehensive decision rules. For MI decision, the ECG signal is shared with the respective cardiologist who analyses and prescribes the required medication to the first-aid professional through the cloud. The first-aid professional is notified accordingly to attend the patient immediately. To avoid the identity crisis, ECG signal is being watermarked and uploaded to the cloud in a compressed form. The proposed system reduces both data storage space and transmission bandwidth which facilitates accessibility to quality care in much reduced cost.
\end{abstract}

\section{KEYWORDS}

Automation, Cloud, ECG, Healthcare, MI, Security, Telemonitoring, Watermarking

\section{INTRODUCTION}

Myocardial infarction (MI), a harmful cardiovascular disease (CVD), is one of the most serious causes of death worldwide, now a days. Every year, nearly 8 million deaths occur globally due to the above disease (WHO, 2017). MI, popularly known as heart attack is responsible for creating a global lifethreatening circumstance. It is a fact that, more than 610,000 people in the USA only get distressed by MI (cdc.gov, 2017) with annual direct estimated costs of over \$316 billion (Benjamin et.al, 2017). In the current scenario, it expands in an epidemic manner and will continuously destroy the heart muscles if not treated timely. So early and accurate detection of MI can improve the diagnoses quality and can effectively reduce the mortality rate in the world. As per the records of different statistical surveys, MI is becoming a major health burden also in India. The 70\% rural population of India who lives in remote villages are found to be harassed in the name of timely accessible medical treatment, because 
of only $2 \%$ of specialist cardiologists are in rural areas. Hence, timely medical treatment for rural populations, reduce the increasing burden of CVD and total medical cost are crucial and demanding.

Cloud-based Telemonitoring service has the prospective strategy to enhance the primary care of CVD, which has the ability to reduce the increasing burden of CVD on healthcare system (Bashi et al, 2017). Telemonitoring services not only support the automatic analysis of medical data but also alert the first-aid professional for any important detectable changes. It has the potential to connect with the cardiologists, who recommended medication to the first-aid professional through the cloud server. The first-aid professional is attended to the patient needs accordingly.

One of the major concerns in the cloud-based Telemonitoring service is the healthcare data security and patient's privacy which will be a great impact for further success of cloud- based healthcare automation system. During cloud transfer or synchronization healthcare data are prone toward hackers with interconnected devices. So, this healthcare data must be protected from any kind of unlawful access. Watermarking technique can play a crucial role to protect healthcare data by combining the confidential information with the healthcare data.

The purpose of watermarking is hiding a message, called watermark, related to a digital signal (i.e. an image, text, song, and video) within the signal itself. Thus in a cloud based Telemonitoring system data security in the form of watermarking and authentication is very important.

The healthcare data produced by monitoring systems sometimes may be voluminous and range for long time period. The Huge amount of bandwidth is needed for the data transmission to the doctor's end. If the data is compressed, then these huge amounts of bandwidth may avoid. Hence an efficient healthcare data compression technique is required to reduce the huge amount of data as much as possible for analysis, storage and transmission (Halder et al, 2014a). The main target of any compression technique is to maximize the data volume reduction during the preservation of significant features and also to detect and eliminate redundancies in a given dataset.

This paper primarily focuses on the development of a primary level architectural framework for healthcare automation system using cloud-based Tele monitoring technique. In this system, the specialist Doctor is not needed in the initial stage. As a case study, ECG signal is used as a significant assessment tool. The patient should first turn up in the healthcare center for ECG testing and recorded ECG sent to the desktop via Bluetooth technology and consequently digitized ECG signal in voltage time format is generated. Recorded ECG signal is being watermarked to avoid the identity crisis and transmitted to the cloud server in a compressed form (Halder \& Mitra, 2015; Halder \& Mitra, 2014b; Halder et al, 2014c) for a longer time. On the other hand, the proposed system removes unnecessary noise from the recorded signal and extracts all the clinically significant features by using Adaptive window dependent differential histogram approach (Halder et al, 2016; Halder et al, 2018). Extracted features further classified by a rule-based rough set classification system. If the classification decision is Non-MI, then the first-aid professional directly reports to the patient. Otherwise, the uploaded signal shares with the respective cardiologist for analysis. Instantly, cardiologist gets a notification mail and a message in his phone. He analyses the downloaded decompressed signal and prescribes the needed medication to the first-aid personal through the cloud without delay. The first-aid personal is immediately notified and attend to the patient needs accordingly. In this research, the online storage cloud Dropbox is used. IFTTT (If this then that) tool is also used to connect the cardiologist phone number with an E-mail account. The suitability of this system is that a rural patient at risk can be constantly monitored without the physical presence of cardiologist and healthcare data sent securely to the cloud for faultless access from anywhere and anytime. Such a system may be used for diagnosis and prediction purposes and also to reduce the increasing burden of CVD and health care costs. This paper is the extension of a previous publication which reported compression, watermarked and feature extraction from the system perspective (Halder et al, 2016; Halder et al, 2018; Halder \& Mitra, 2015; Halder \& Mitra, 2014b; Halder et al, 2014c; Halder et al, 2014a). In accordance with the available information, this is the first study that designed a cloud-based Telemonitoring technique for healthcare automation system with MI classification. 
The rest of the paper is arranged as follows: Section 2 reports an overview of related works in cloud-based telemonitoring. Section 3 briefly describes the architectural design aspect of the proposed Healthcare Automation system. Synchronizing the Doctor's phone number with E-mail account using IFTTT is described in Section 4 and Section 5 concludes the paper.

\section{RELATED WORK}

In the last decade, the cloud-based Tele-monitoring technology has been increased for automation of healthcare system and its influences on the activities of healthcare professionals, patients, and the healthcare industry. Similar technologies have been used in the automation of health care system in various works. The booming uses of cloud-based healthcare and biomedicine are already reported (Lin et al, 2013; Lin et al, 2014; Ekanayake et al, 2011; Ahuja et al. 2012). Apart academic researchers, several renowned world-class software companies like Microsoft HealthVault (Calabrese \& Cannataro, 2015) have invested heavily in the cloud.

Aranki et. al (2016) conducted a Telemonitoring system based on a Smartphone to collect the estimates of EE data continuously. After performing the data analysis of daily collected self-reported essential signs and cardiovascular symptoms, it gives alerts to the medical staff. In the suburb of China with 1.1 million populations, a cloud-based HER (electronic health record) system has been executed [Lin et al, 2014]. In this approach, the care provision for ViDs (village doctors) in rural China got improved and the competence of the healthcare system to monitor the health condition of the population has also increased. Using web services and cloud computing, a monitoring system for a remote patient is developed (Mohammed et al, 2014), particularly through an Android application, designed for the monitoring and analysis of ECG data. An autonomic Cloud environment has been proposed in Pandey et al (2012) which collected people's health data and stored them in a Cloudbased information warehouse and facilitates analysis of the data using software services hosted in the Cloud. An expert diagnosis system based on cloud computing has been reported in Tseng, \& $\mathrm{Wu}$. (2014) which classified one's fitness level as per supervised machine learning methods. This system is competent to study and customized diagnoses according to the user's physiological data. Mouleeswaran and colleagues has been proposed to secure open cloud architecture (Open Cloud Care) for remote patient health monitoring (Mouleeswaran et al, 2012). In Chow et al (2009), the use of one of the most reliable computing and applied cryptographic techniques to safe data in the Cloud has been described. In Dey et al (2017), authors reported an optimal data security method to implant a furtive message into the open content. Several methods have been reported (Wang, et al. 2016; Liji et al, 2016) for protection secret patient information and to give authentication data of examination staff and equipment. A point-of-care telemetric sensor technique (Ibaida et al, 2013) has been implemented for provides stegano ECG. This system is diagnosable for every reader to access individual data needs authorization. However, none of the reviewed studies performed the Cloudbased telemonitoring with myocardial infarction (MI) disease classification, ECG signal watermarked and compression together.

To date, this the first study on cloud-based healthcare automation system, where:

1. ECG signal is watermarked and compressed before uploaded to the cloud to authenticate any attacks and transmitting faster;

2. Extracts feature from ECG signal and classify the signal for MI detection before transmitted to the cloud to assist first-aid personal for preliminary analysis decision and patient care immediately;

3. The system has also been decompressed the downloaded signal from doctor's end, analysis the signal and send back a decision and prescription to the first-aid professional through the cloud. The first-aid professional notifies to the patients accordingly. 
In this system first-aid professional may be considered as learner whereas specialist Doctors be the teacher. Moreover, as the whole system is cloud based so it may be compared as a web based technology.

\section{PROPOSED HEALTHCARE AUTOMATION FRAMEWORK}

This section, architectural design aspect of a Cloud-based Telemonitoring technique is described. This entire system takes place between a patient and a doctor via a primary health care center, which connects the two with the help of cloud. The processing steps followed for the proper functioning of proposed ECG monitoring and analysis system are watermark, compression, decompression, feature extraction and classification. Watermarked compressed ECG signal stored in the cloud waiting to be monitored, retrieved and reviewed in worldwide.

\subsection{Architectural Design Aspects}

An illustration of the infrastructure and architectural model for Healthcare Automation system is shown in Figure 1.

The overall functionality of cloud-based Healthcare Automation system involves the following steps:

1. The patient goes to the health center or hospital and registers as a new patient. Then, he undergoes an ECG test via portable ECG machine, sent to the desktop via Bluetooth technology and consequently digitized ECG signal shown in Figure 2 is generated;

2. The software then embeds a watermark and compressed the ECG (discussed in section 3.3). The watermarked and compressed ECG signal then stored in the patient's folder in the health center's DROPBOX (Cloud) account for high-quality health monitoring in the future;

Figure 1. System architecture for new patient's of the rural health care system

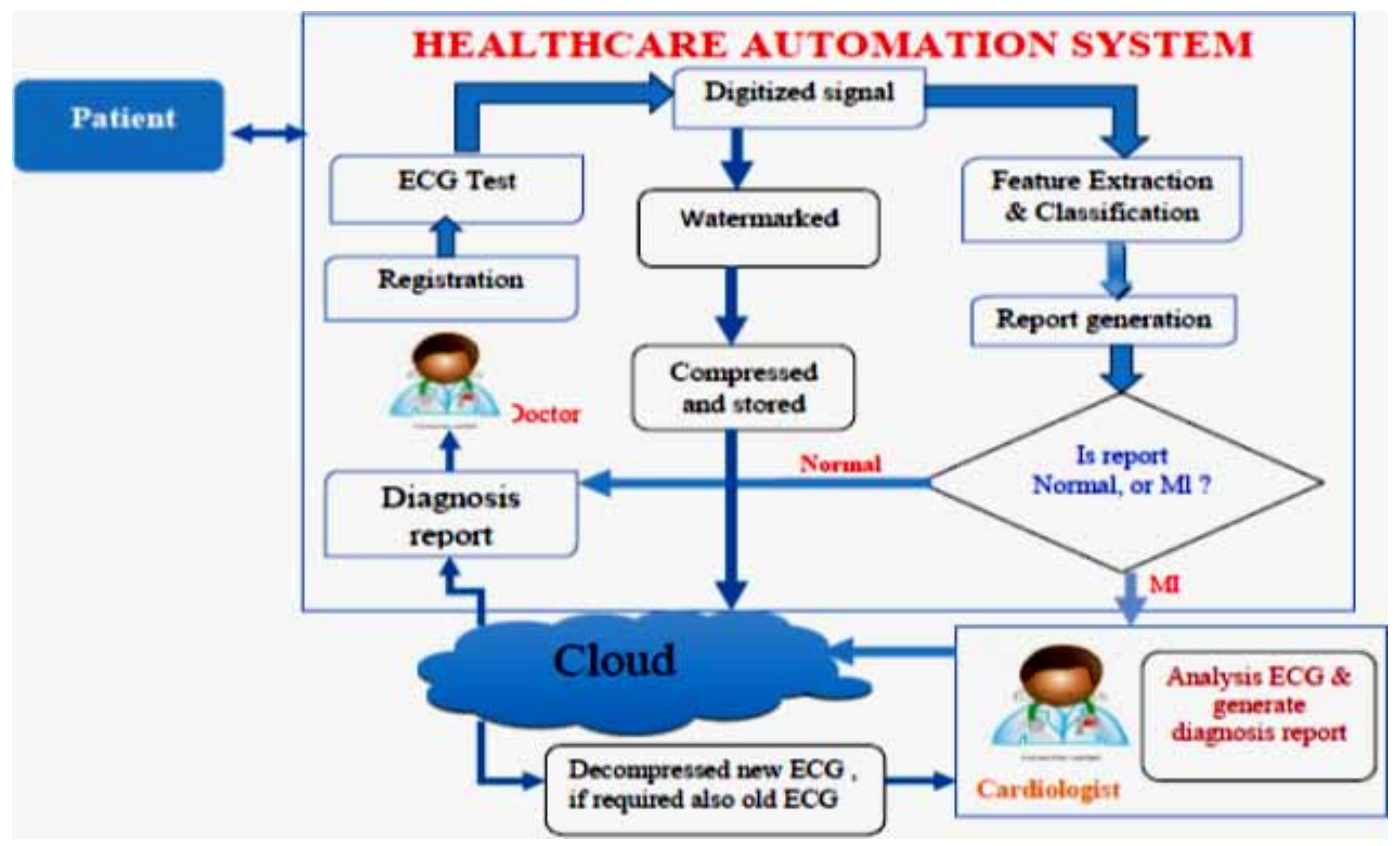


Figure 2. Recorded and filtered ECG signal on patient's end

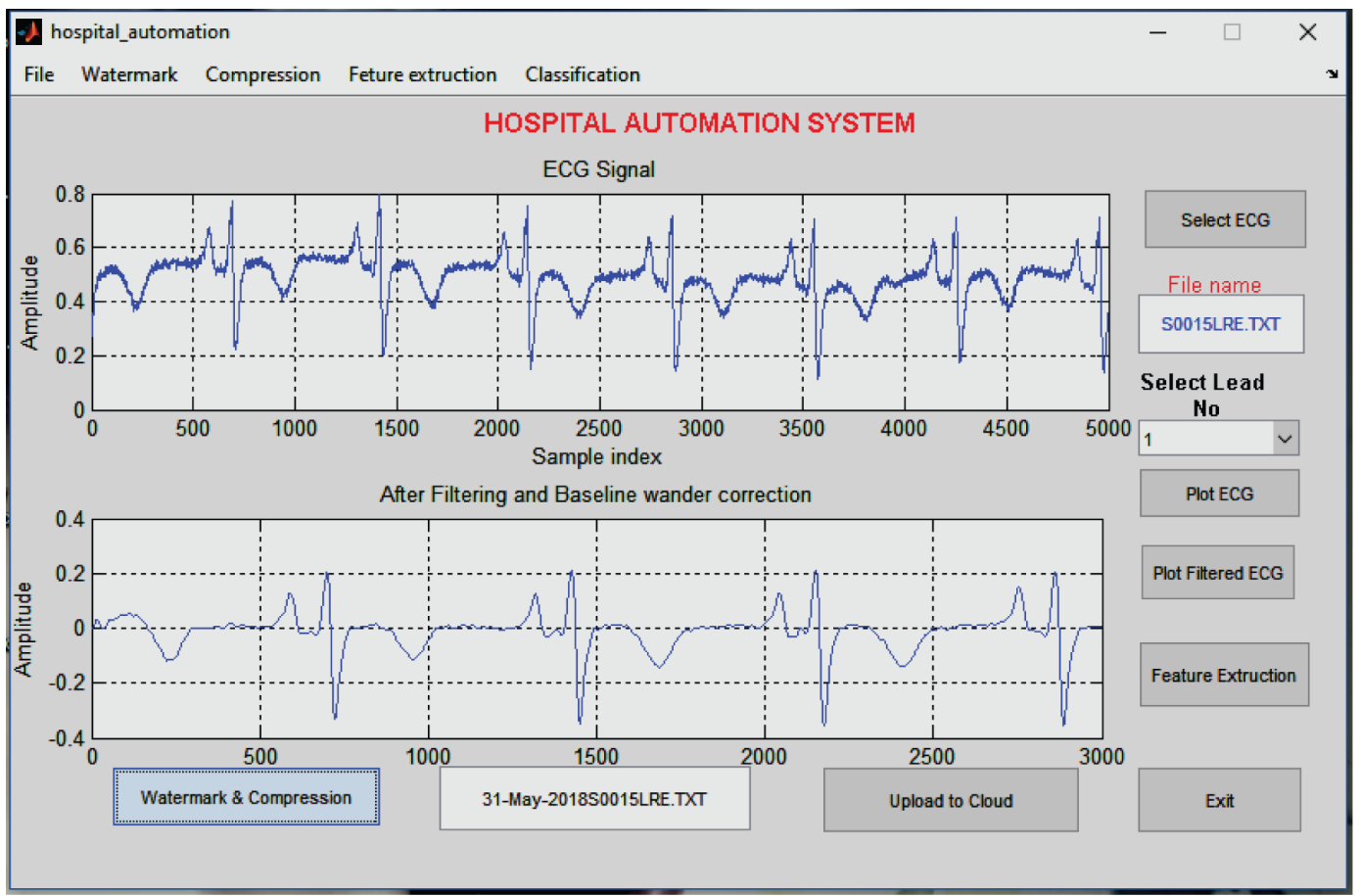

3. On the other hand, the feature extraction software (discussed in sec 3.4) in the system is executed for automatic detection and identification of crucial characteristic features from the recorded ECG signal and medically significant interval and duration within a very short period is also determined;

4. The classification software then (discussed in sec 3.5) used for detection of myocardial infarction (MI) diseases over the extracted clinically significant characteristic of ECG signal;

5. If the classification decision is MI, then uploaded compressed ECG file shares with the respective Cardiologist. The Cardiologist gets a notification mail and a message on his phone instantly;

6. The cardiologist immediately opened this system and click on the "Download from cloud" button to go to the file. Then, the cardiologist can visualize the file shared by the first-aid personal and click on the "Open download file" button to view it (shown in Figure 3);

7. The cardiologist analyses the downloaded decompressed signal shown in Figure 3 and writes the prescription for the patient in the comment section of the cloud. The first-aid personal is immediately notified of the cardiologist's comment and attend to the patient needs accordingly;

8. The first-aid professional can reply to the cardiologist's comment by clicking on the "reply" link in the comment section shown in Figure 4. Once, the first-aid personal comments on it, the cardiologist gets notified instantly both via mail and message. The cardiologist can then click on the notification and reply accordingly;

9. If the patient is an old patient, the software then decompresses his old records from the cloud; the Cardiologist then compares the old record with the new report and prescribes the needed medication and analysis report to the first-aid professional.

Thus, this procedure continues until the patient is diagnosed for the time being. When the patient again comes to the healthcare center his previous records are again collected in the manner mentioned above and shares with the cardiologist. The proposed system decompressed the previous records and 
Figure 3. Downloaded decompressed signal on doctor's end

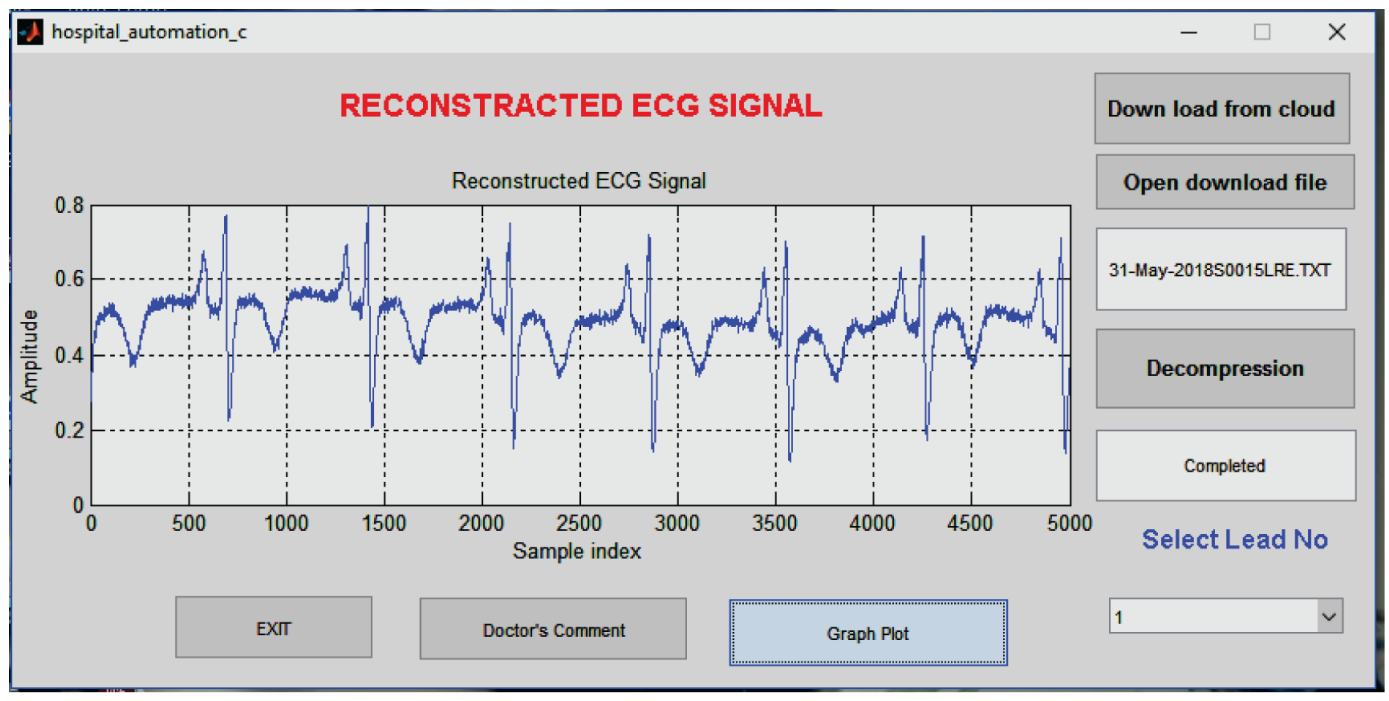

Figure 4. Screenshot of the first-aid personal and doctor's comments

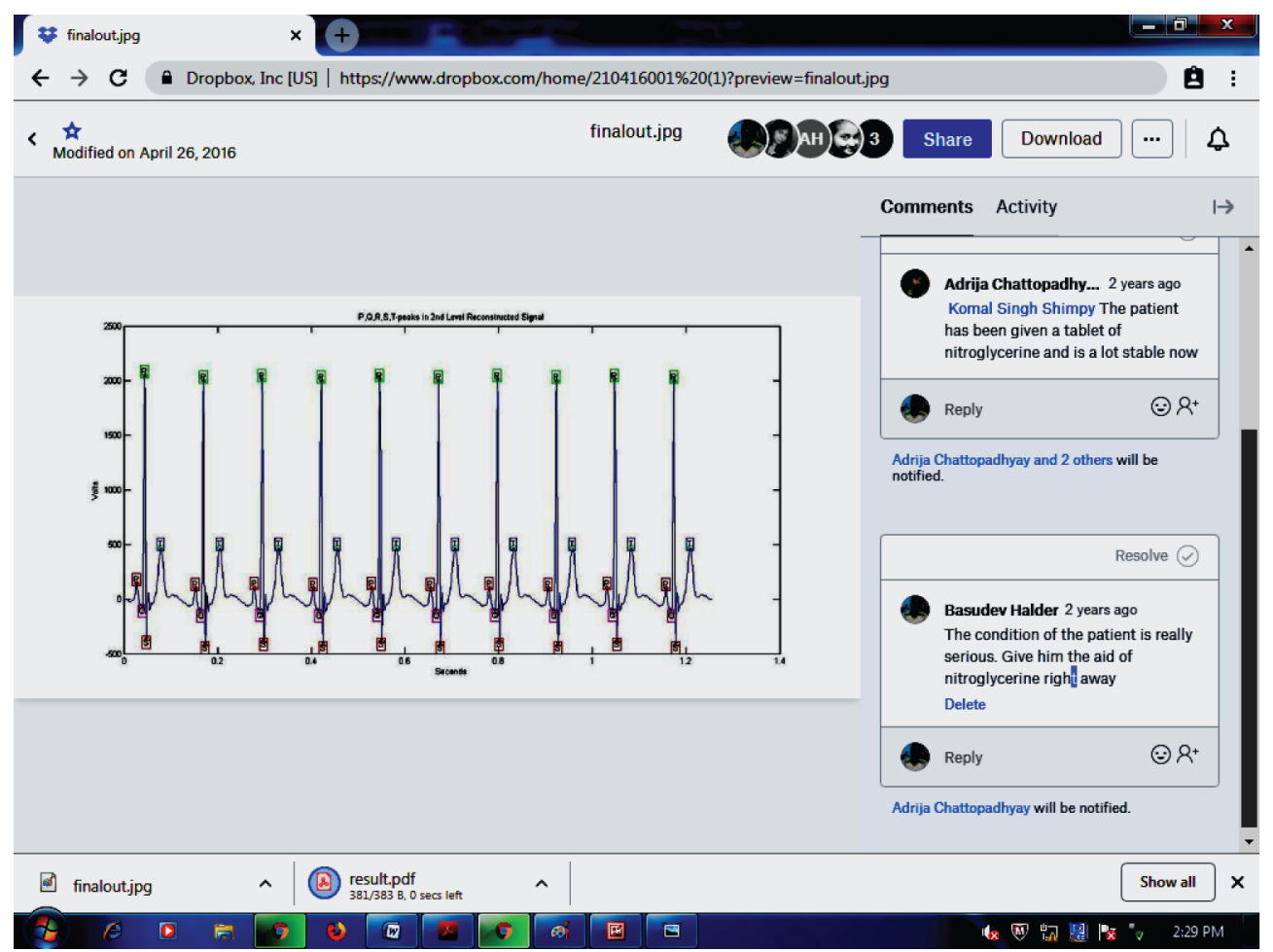


uploads them in the folder to be compared with the patient's present status. The Cardiologist gets notified and he checks both the records and comments again.

\subsection{Data Analytics}

The architecture as shown in Figure 5 explains how the doctor analytics both the patient's decompressed old records and the new records as shared by the first-aid professional in the doctor's Dropbox account. The doctor is first notified of the patient's folder. The overall functionality of data analytics system involves the following steps:

1. The software decompressed patient's old records from the cloud;

2. The Cardiologist is first notified of the patient's folder. He then analytics the old record with the new record and then suggest proper medication for the patient;

3. The diagnosis report that the cardiologist makes is uploaded to the cloud;

4. The first-aid professional then view it and meets the medical needs of the patients.

\subsection{ECG Watermarked and Compression}

In this paper, diagnosis report is watermarked with patient information using LSB watermark technique and compressed the huge amount of data using ASCII character encoding in order to confirm the patient integrity and reduced the bandwidth. The basic idea of the watermarking and compression technique is described in our previous published paper (Halder et al, 2016; Halder et al, 2018; Halder \& Mitra, 2015; Halder \& Mitra, 2014b; Halder et al, 2014c; Halder et al, 2014a).

\subsection{Feature Extraction}

In this paper, Differential histogram approach depends on an Adaptive window has been used for take outing all pathological features from ECG signals which may further be used to identify the cardiac difficulty. The basic idea of this technique is described in our previous published paper (Halder et al, 2016; Halder et al, 2018). The obtained differential histogram of an ECG signal is shown in Figure 6. The entire clinically significant feature extracted by this technique is shown in Figure 7. The time duration and different amplitude of characteristic points have been computed and tabulated in Table 1. The performance of the Histogram based ECG detector is found satisfactory with $99.52 \%$ average accuracy of the QRS detector.

\subsection{Development of Basic Knowledge and MI Detection System}

We have also emphasized on the analysis and classification of cardiac disease. The rule-based rough set classifier is developed using RSES tool (Bazan \& Szczuka, 2005; Øhrn, 2000) for myocardial

Figure 5. Analytics workflow architecture

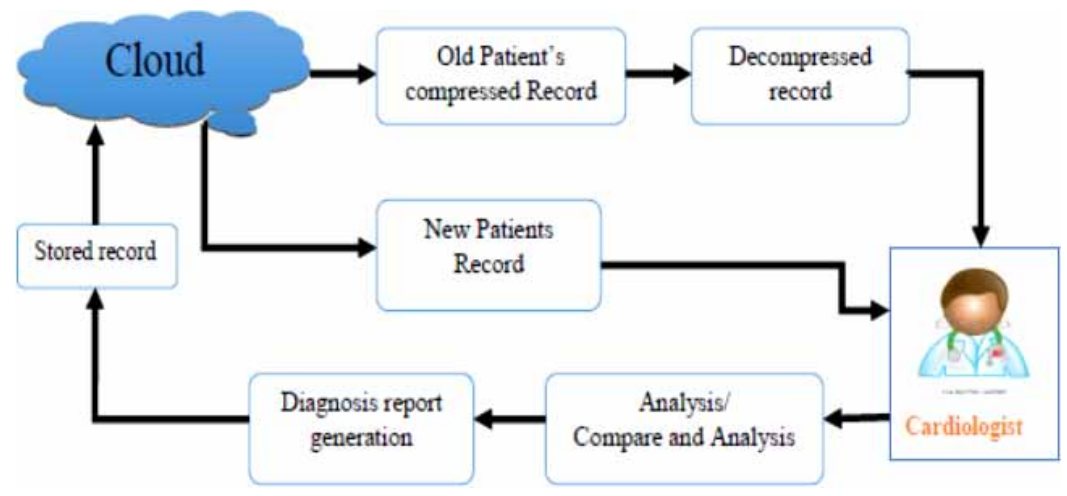


Figure 6. Detection of $P$ peak, $R$ peak and T peak region of an ECG signal by differential histogram

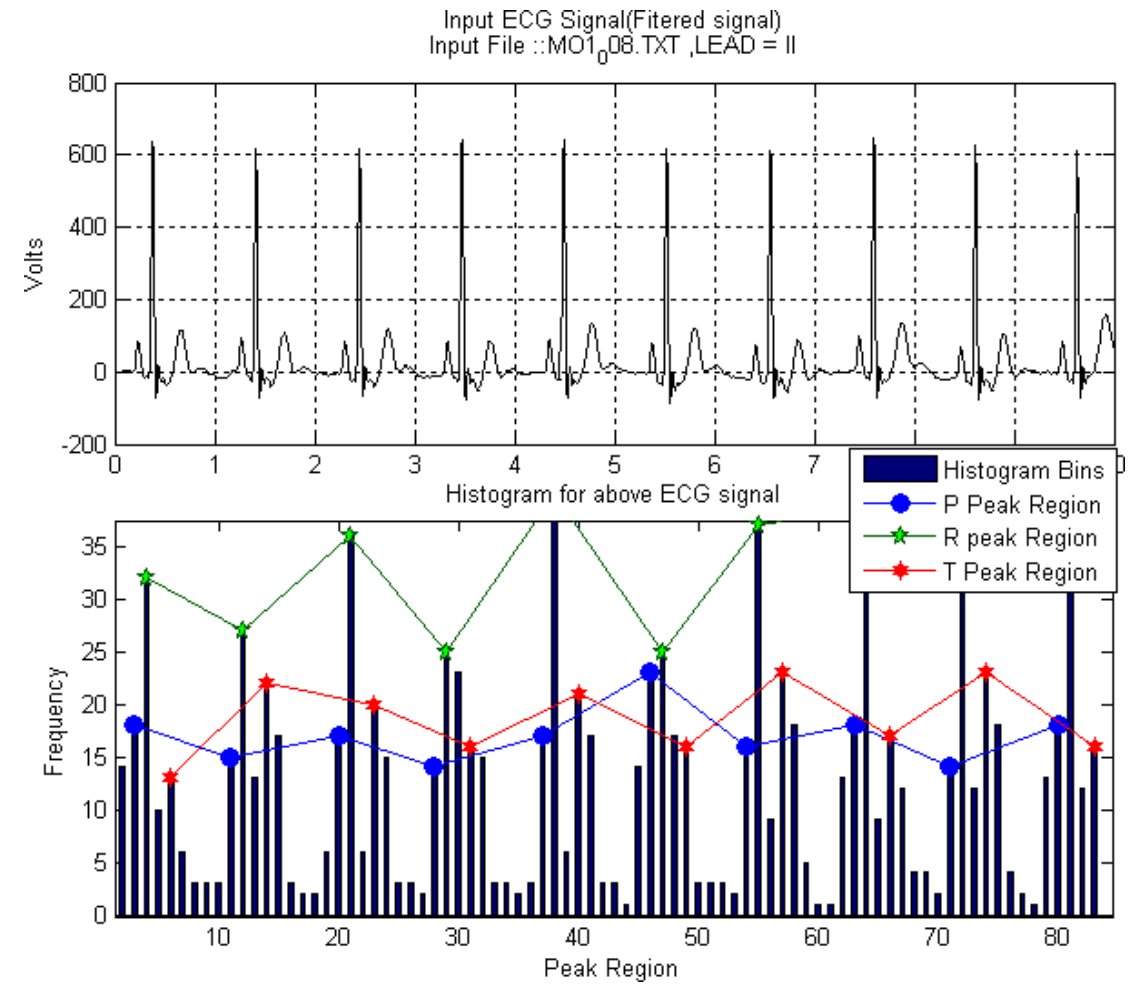

Figure 7. Recognition of pathological features of an ECG signal by histogram method

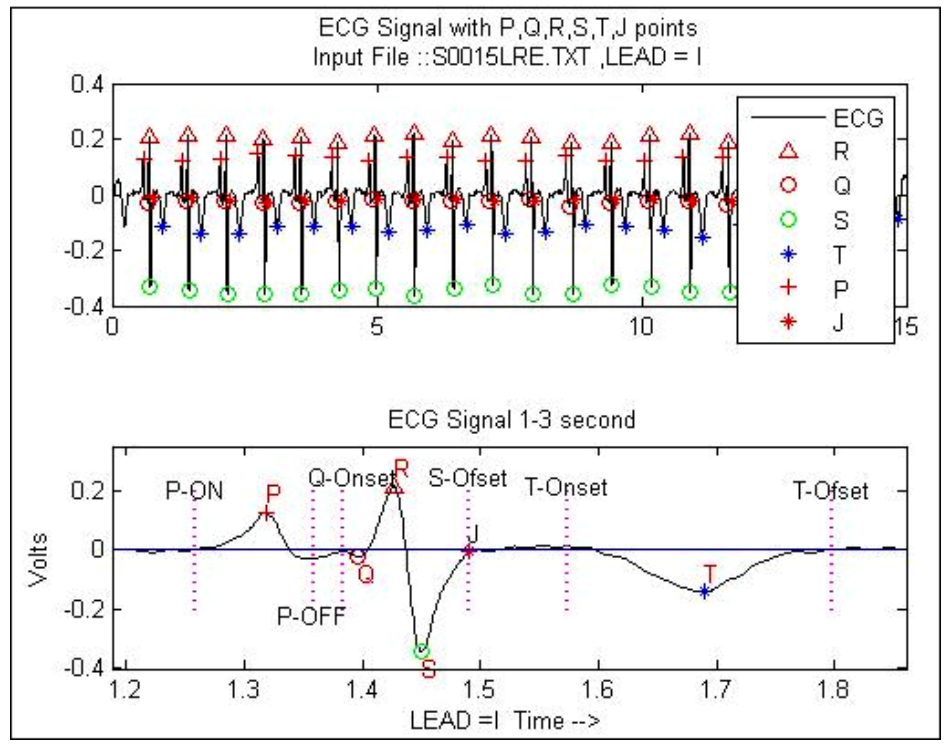


Table 1. Pathological features of ECG signal

\begin{tabular}{|c|c|c|c|c|c|c|c|c|c|c|c|}
\hline \multirow{2}{*}{ File Name } & \multirow{2}{*}{$\begin{array}{l}\text { Lead } \\
\text { No. }\end{array}$} & HR & $\begin{array}{c}\text { QRS } \\
\text { Duration }\end{array}$ & $\begin{array}{c}R \\
\text { Height }\end{array}$ & $\begin{array}{c}\mathbf{P} \\
\text { Duration }\end{array}$ & $\begin{array}{c}\text { P } \\
\text { Height }\end{array}$ & $\begin{array}{c}T \\
\text { Height }\end{array}$ & $\begin{array}{c}\text { PR } \\
\text { Interval }\end{array}$ & $\begin{array}{c}\text { QT } \\
\text { Interval }\end{array}$ & $\begin{array}{c}\text { ST } \\
\text { Segment }\end{array}$ & QTe \\
\hline & & bps & ms & $\mathbf{m v}$ & ms & $\mathbf{m v}$ & $\mathbf{m v}$ & ms & ms & ms & ms \\
\hline MO1_125 & V6 & 72 & 95 & 1.0863 & 88 & 0.05 & 0.26 & 113 & 388 & 122 & 434 \\
\hline MA1_004 & I & 72 & 104 & 0.5399 & 75 & 0.03 & 0.13 & 111 & 389 & 116 & 416 \\
\hline MA1_004 & II & 72 & 106 & 0.8412 & 115 & 0.05 & 0.16 & 144 & 387 & 114 & 407 \\
\hline MA1_125 & 1 & 78 & 106 & 0.4298 & 106 & 0.07 & 0.18 & 119 & 404 & 31 & 434 \\
\hline MO1_099 & I & 60 & 92 & 0.5759 & 96 & 0.06 & 0.36 & 126 & 384 & 121 & 382 \\
\hline MO1_099 & II & 60 & 94 & 0.4987 & 102 & 0.07 & 0.34 & 137 & 386 & 120 & 390 \\
\hline MO1_008 & V6 & 60 & 94 & 1.3136 & 87 & 0.05 & 0.21 & 130 & 408 & 143 & 403 \\
\hline MO1_043 & I & 60 & 97 & 0.46 & 102 & 0.05 & 0.21 & 112 & 408 & 141 & 416 \\
\hline MO1_043 & II & 60 & 92 & 0.86 & 96 & 0.00 & 0.09 & 145 & 410 & 157 & 418 \\
\hline MO1_043 & aVF & 60 & 89 & 0.66 & 90 & 0.00 & 0.03 & 153 & 418 & 219 & 426 \\
\hline
\end{tabular}

infarction (MI) disease detection. For this purpose, a knowledgebase shown in Table 2 is expanded from the derived features of ECG signal after the feedback from reputed cardiologists and consultation of different medical books (Schamroth, 2009; Goldberger, 2002) as well. We have selected 7 times plane features (attributes) shown in Table 3 for disease detection. Based on the different pathological features or different values of attributing the decision of the sample may be either MI or non-MI data.

In this research, we have considered two types of MI: Anterior (AN) and Inferior (IN). When infarction or necrosis happens in the Inferior wall of the heart then it is classified as Inferior infarction and it is diagnosed from ECG lead II, III and aVF. Similarly, infarction in Anterior wall show the signature of Anterior infarction and diagnosed leads are V2, V3 and V4. The pathological features such as inverted T wave, elevated ST segment or pathological Q wave shown in Table 2 is investigative of MI. These sets of pathological features are used as an information system shown in Table 4 for classification. In an information system, some conditional attributes may be redundant and can be eliminated without losing essential information. Rule based Rough set classifier (Halder et al, 2019) allows us to determine a degree of attributes dependency and their importance to find a smaller set of attributes, called minimal reduct, with the same as the original set and forecasted the universal decision rules to identify $\mathrm{MI}$.

Let $\mathrm{A}=(\mathrm{U}, \mathrm{A})$ be an information system and let $\mathrm{X}$ (Decisional attribute) and $\mathrm{Y}$ (conditional attribute) be subsets of $\mathrm{A}$ and $\mathrm{Z}$ be subset of $\mathrm{U}$. Decisional attribute $(\mathrm{X})$ is depends totally on conditional attribute $(\mathrm{Y})$, denoted $\mathrm{Y} \Rightarrow \mathrm{X}$, if all the decisional attribute $(\mathrm{X})$ are uniquely determined by the value of conditional attribute $\mathrm{Y}$. Degree of dependency of attribute $\mathrm{X}$ on attribute $\mathrm{Y}$, denoted $Y \Rightarrow_{p} X$ is defined by:

Table 2. A knowledgebase for cardiac diseases

\begin{tabular}{|l|l|l|l|l|l|}
\hline $\begin{array}{c}\text { Localization } \\
\text { Wall }\end{array}$ & \multicolumn{1}{|c|}{$\begin{array}{c}\text { Elevated ST } \\
\text { Segment }\end{array}$} & $\begin{array}{c}\text { Depressed ST } \\
\text { Segment }\end{array}$ & $\begin{array}{c}\text { Inverted T } \\
\text { Wave }\end{array}$ & Upright T Wave & Pathologic Q Wave \\
\hline Anterior MI & V2,V3,V4 & II,III,VF & V2,V3,V4 & II,III,VF & V2,V3,V4 \\
\hline Inferior MI & II,III,VF & I,VL & II,III,VF & I,VL & II, III,VF \\
\hline
\end{tabular}


Table 3. Description of the attributes in the MI database

\begin{tabular}{|l|l|l|}
\hline \multicolumn{1}{|c|}{ SL No. } & \multicolumn{1}{c|}{ Attributes Name } & \multicolumn{1}{c|}{ Attribute Feature } \\
\hline 1 & ST segment & Elevated, Depressed, ISO electric \\
\hline 2 & T Wave & Inverted, Upward \\
\hline 3 & Q Pathologic & Yes, No \\
\hline 4 & HR (bpm) & Normal, Abnormal \\
\hline 5 & QRS width (sec) & Normal, Abnormal \\
\hline 6 & R height (mv) & Normal, Abnormal \\
\hline 7 & QTc (sec) & Normal, Abnormal \\
\hline
\end{tabular}

Table 4. A portion of the decision table, here N: normal, AN: Anterior, IN: Inferior

\begin{tabular}{|c|c|c|c|c|c|c|c|c|c|c|c|c|c|c|c|c|c|c|c|c|c|c|c|c|c|c|c|c|c|c|c|c|c|c|c|c|}
\hline $\begin{array}{l}z \\
\bar{z}\end{array}$ & है & $\begin{array}{c}- \\
0 \\
\frac{3}{3} \\
3 \\
- \\
- \\
\end{array}$ & 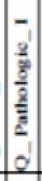 & $\begin{array}{l}\frac{5}{5} \\
\frac{5}{5}\end{array}$ & $\begin{array}{l}= \\
= \\
\frac{3}{3} \\
3\end{array}$ & $\begin{array}{l}= \\
\frac{3}{2} \\
\frac{2}{2} \\
\frac{2}{2} \\
\frac{2}{2} \\
\frac{2}{2} \\
0 \\
\end{array}$ & 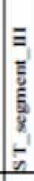 & $\begin{array}{l}\equiv \\
\vdots \\
3 \\
3 \\
3\end{array}$ & 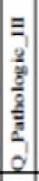 & $\mid \begin{array}{c}5 \\
5 \\
\frac{1}{5} \\
\frac{5}{5} \\
\frac{5}{2} \\
5 \\
5\end{array}$ & & $\begin{array}{l}\frac{5}{2} \\
\frac{2}{2} \\
\frac{2}{2} \\
\frac{8}{2}\end{array}$ & 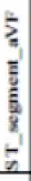 & $\begin{array}{l}5 \\
5 \\
3 \\
3 \\
3 \\
3\end{array}$ & 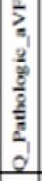 & $\mid \begin{array}{c}5 \\
5 \\
\frac{5}{5} \\
\frac{5}{3} \\
\frac{8}{1} \\
5 \\
\end{array}$ & 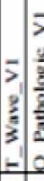 & $\frac{2}{6}$ & 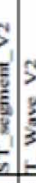 & $=\begin{array}{l}5 \\
\frac{2}{2} \\
\frac{2}{2} \\
\frac{2}{2} \\
0 \\
0\end{array}$ & $\begin{array}{l}5 \\
\frac{5}{5} \\
\frac{5}{3} \\
5\end{array}$ & 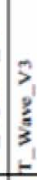 & $\begin{array}{l}5 \\
\frac{2}{2} \\
\frac{8}{2} \\
\frac{2}{2} \\
0\end{array}$ & 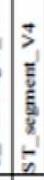 & 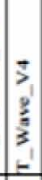 & $\mid \begin{array}{l}5 \\
1 \\
\frac{1}{2} \\
\frac{5}{0} \\
\frac{2}{2} \\
\frac{2}{2} \\
0\end{array}$ & $\begin{array}{l}\frac{1}{5} \\
\frac{1}{3} \\
0\end{array}$ & $\begin{array}{l}3 \\
\vdots \\
\vdots \\
\vdots \\
\vdots \\
\vdots \\
\vdots\end{array}$ & $\begin{array}{l}3 \\
\frac{n}{1} \\
\frac{2}{0} \\
\frac{5}{2} \\
\frac{2}{2} \\
\frac{2}{2} \\
0 \\
0\end{array}$ & $\begin{array}{l}\frac{5}{2} \\
\frac{5}{5} \\
\frac{1}{5} \\
5 \\
0\end{array}$ & $\begin{array}{l}0 \\
3 \\
3 \\
0 \\
3 \\
3 \\
3 \\
1\end{array}$ & $\begin{array}{l}\frac{0}{1} \\
\frac{1}{2} \\
\frac{2}{2} \\
\frac{2}{2} \\
\frac{2}{2} \\
0\end{array}$ & 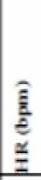 & $\begin{array}{l}\hat{y} \\
\frac{8}{3} \\
\frac{y}{3} \\
\frac{2}{2} \\
\frac{2}{2}\end{array}$ & $\begin{array}{l}8 \\
3 \\
0 \\
0 \\
\end{array}$ & $\begin{array}{l}\hat{\theta} \\
0 \\
0 \\
0 \\
0 \\
0\end{array}$ \\
\hline 14 & iso & $\mathrm{v}$ & $\mathrm{N}$ & $D$ & U & $\mathrm{N}$ & D & $\mathrm{U}$ & $\mathbb{N}$ & iso & $\mathrm{U}$ & $N$ & $D$ & U & $\mathrm{N}$ & iso & $\begin{array}{lll}0 & 1\end{array}$ & $N$ & $\begin{array}{l}E \\
\end{array}$ & 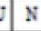 & $\mathrm{E}$ & $v$ & $\mathbb{N}$ & $E$ & $v$ & $\mathbb{N}$ & $\bar{E}$ & $\mathrm{v}$ & $\mathrm{N}$ & $E$ & $\mathrm{v}$ & $\mathrm{N}$ & 100 & 100 & No & $\mathrm{N}$ \\
\hline 15 & $\mathrm{E}$ & $\mathrm{v}$ & $\mathrm{N}$ & iso & v & $\mathrm{N}$ & iso & 0 & \begin{tabular}{|l|}
$\mathrm{N}$ \\
\end{tabular} & \begin{tabular}{|l|l|} 
iso \\
\end{tabular} & 1 & $\mathrm{~N}$ & 50 & 0 & \begin{tabular}{|l|}
$\mathrm{N}$ \\
\end{tabular} & \begin{tabular}{|l|} 
iso \\
\end{tabular} & 01 & N & $E 0$ & $0 \mathrm{~N}$ & $\bar{E}$ & $\mathrm{v}$ & $\mathrm{N}$ & $\mathrm{E}$ & $\mathrm{v}$ & $\mathrm{N}$ & $\mathrm{E}$ & $\mathrm{v}$ & $\mathrm{N}$ & $\mathrm{E}$ & $\mathrm{U}$ & $\mathrm{N}$ & \begin{tabular}{|l|} 
No \\
\end{tabular} & 10 & No & $\mathrm{N}$ \\
\hline 16 & iso & $\mathrm{v}$ & $\mathrm{N}$ & 130 & U & N & D & $\mathrm{U}$ & $\mathrm{Y}$ & $\mathrm{E}$ & $\mathrm{U}$ & $\mathrm{N}$ & D & $\mathrm{U}$ & $\mathrm{N}$ & iso & \begin{tabular}{|l|l}
1 & $B$ \\
\end{tabular} & N & $\begin{array}{ll}E & 0 \\
\end{array}$ & $\mathbb{N}$ & $\mathrm{E}$ & U & $\mathrm{N}$ & E & $\mathrm{v}$ & $\mathrm{N}$ & $\mathrm{E}$ & $\mathrm{v}$ & $\mathrm{N}$ & so & $\mathrm{U}$ & $N$ & No & 10 & No & $\mathrm{N}$ \\
\hline 17 & $\mathrm{E}$ & $\mathrm{v}$ & $\mathrm{N}$ & iso & $\mathrm{U}$ & $\mathbb{N}$ & D & U & $\mathrm{N}$ & $\mathrm{E}$ & $\mathrm{v}$ & $\mathrm{N}$ & iso & $\mathrm{U}$ & $\mathrm{N}$ & iso & $\begin{array}{lll}\mathrm{O} & \mathrm{S}\end{array}$ & $\mathrm{N}$ & is 1 & $\mathbb{N}$ & iso & U & $\mathrm{N}$ & iso & $\mathrm{v}$ & $\mathrm{N}$ & $\mathbf{E}$ & $\mathrm{v}$ & $\mathrm{N}$ & iso & $\mathrm{v}$ & $\mathbb{N}$ & No & 100 & No & $\mathrm{N}$ \\
\hline 18 & $\mathrm{E}$ & $\mathrm{U}$ & $\mathrm{N}$ & E & U & $\mathbb{N}$ & E & $\mathrm{U}$ & $\mathbb{N}$ & $E$ & U & $Y$ & $\mathrm{E}$ & U & $\mathbb{N}$ & iso & $\begin{array}{lll}0 & 1 \\
\end{array}$ & $\mathrm{Ni}$ & 500 & 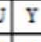 & iso & $\mathrm{v}$ & $Y$ & iso & $\mathrm{v}$ & $\mathrm{N}$ & iso & $\mathrm{v}$ & $\mathrm{N}$ & is & $\mathrm{U}$ & $\mathbb{N}$ & 100 & 10 & No & $N$ \\
\hline 19 & $\mathbf{E}$ & $\mathrm{U}$ & $\mathrm{N}$ & D & $\mathrm{U}$ & \begin{tabular}{|l|}
$\mathbb{N}$ \\
\end{tabular} & D & $\mathrm{v}$ & \begin{tabular}{|l|}
$\mathbb{N}$ \\
\end{tabular} & iso & $\mathrm{U}$ & $\mathbb{N}$ & $D$ & $\mathrm{U}$ & $\mathrm{N}$ & $E$ & 0.1 & $\mathrm{~N}$ & $E U$ & $\mathbb{N}$ & E & $\mathrm{U}$ & $\mathrm{N}$ & $\mathbf{E}$ & $\mathrm{v}$ & $\mathbb{N}$ & iso & 0 & $\mathrm{~N}$ & bo & $\mathrm{v}$ & $\mathbb{N}$ & 10 & No & No & $\mathrm{N}$ \\
\hline 20 & $\mathrm{E}$ & I & $\mathrm{N}$ & $D$ & U & N & D & $\mathrm{U}$ & \begin{tabular}{|l|}
$N$ \\
\end{tabular} & $E$ & I & $\mathbb{N}$ & $\mathrm{D}$ & $\mathrm{v}$ & $\mathrm{Y}$ & $E$ & U 1 & $\mathrm{~N}$ & EII & 7 & $\mathrm{E}$ & I & $\mathrm{Y}$ & $E$ & $\mathrm{I}$ & $\mathrm{Y}$ & E & $\mathrm{I}$ & $N$ & $E$ & $\mathrm{v}$ & $\mathbb{N}$ & 100 & 10 & 100 & $A \mathbb{N}$ \\
\hline 21 & $D$ & \begin{tabular}{|l|}
$\mathrm{I}$ \\
\end{tabular} & \begin{tabular}{|l|}
$N$ \\
\end{tabular} & D & 0 & \begin{tabular}{|l|}
$N$ \\
\end{tabular} & D & $\mathrm{U}$ & \begin{tabular}{|l|}
$N$ \\
\end{tabular} & $\mathrm{E}$ & \begin{tabular}{|l|l|} 
I \\
\end{tabular} & $\mathbb{N}$ & D & 0 & \begin{tabular}{|l|}
$\mathrm{N}$ \\
\end{tabular} & $\mathrm{E}$ & \begin{tabular}{|l|l|l|} 
I & P \\
\end{tabular} & N & \begin{tabular}{l|l}
$E$ & 1
\end{tabular} & $\begin{array}{lll}N \\
\end{array}$ & $E$ & 1 & $\mathbb{N}$ & $E$ & $\mathrm{I}$ & \begin{tabular}{|l|}
$\mathrm{N}$ \\
\end{tabular} & $\mathrm{E}$ & $\mathrm{I}$ & $\mathrm{N}$ & $\mathrm{E}$ & $\mathrm{U}$ & $\mathrm{N}$ & 100 & 100 & 100 & AN \\
\hline 22 & $\mathbf{E}$ & I & $\mathbb{N}$ & D & U & N & D & U & $\mathrm{Y}$ & $\mathrm{E}$ & I & $\mathrm{N}$ & D & 0 & $\mathrm{Y}$ & iso & U 1 & 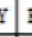 & $E t$ & $0 \mathrm{~N}$ & E & I & $\mathbb{N}$ & E & I & $\mathrm{N}$ & $\mathrm{E}$ & $\mathrm{I}$ & $\mathrm{N}$ & $\mathrm{E}$ & $\mathrm{U}$ & $\mathbb{N}$ & No & 10 & No & AN \\
\hline 23 & $D$ & U & $\mathrm{Y}$ & D & 0 & \begin{tabular}{|l|}
$N$ \\
\end{tabular} & D & $\mathrm{U}$ & \begin{tabular}{|l|}
$\mathrm{N}$ \\
\end{tabular} & \begin{tabular}{|l|}
$E$ \\
\end{tabular} & $\mathrm{I}$ & $\mathrm{N}$ & D & $\mathrm{v}$ & $\mathrm{N}$ & $\mathrm{E}$ & $0 \mathrm{~N}$ & N & \begin{tabular}{|ll}
$E$ & 1
\end{tabular} & $\mathbb{N}$ & $E$ & 1 & $\mathbb{N}$ & $\mathrm{E}$ & 1 & $\mathrm{~N}$ & $\mathbf{E}$ & 1 & $\mathrm{~N}$ & $\mathbf{E}$ & 0 & $N$ & No & 100 & No & AN \\
\hline 24 & $\mathbf{E}$ & I & $\mathrm{N}$ & D & $\mathrm{U}$ & N & D & $\mathrm{v}$ & \begin{tabular}{|l|}
$\mathrm{N}$ \\
\end{tabular} & $\mathrm{E}$ & I & $\mathrm{N}$ & D & $\mathrm{v}$ & $\mathbb{N}$ & $\mathrm{E}$ & \begin{tabular}{l|l}
$\mathrm{I}$ \\
\end{tabular} & N & $\begin{array}{lll}E & 1\end{array}$ & $\mathbb{N}$ & $\mathrm{E}$ & 1 & $\mathrm{Y}$ & E & I & $Y$ & D & $v$ & $\mathrm{Y}$ & $\mathrm{E}$ & 0 & $\mathrm{~N}$ & 100 & 100 & No & AN \\
\hline 25 & $\mathrm{E}$ & I & $\mathrm{Y}$ & $D$ & 0 & N & D & $\mathrm{U}$ & $\mathrm{N}$ & $\mathrm{E}$ & I & $\mathrm{N}$ & D & 0 & $\mathrm{~N}$ & iso & U & $\begin{array}{l}4 \\
\end{array}$ & $\begin{array}{ll}E 1 \\
\end{array}$ & $\mathbb{N}$ & E & I & $\mathrm{N}$ & E & $\mathrm{I}$ & $\mathrm{N}$ & $\mathrm{E}$ & $\mathrm{I}$ & $\mathrm{N}$ & $\mathrm{E}$ & $\mathrm{U}$ & $\mathrm{N}$ & 100 & 100 & No & AN \\
\hline 26 & $D$ & $\mathrm{U}$ & $\mathrm{N}$ & $E$ & 1 & \begin{tabular}{|l|}
$N$ \\
\end{tabular} & $\mathrm{E}$ & I & \begin{tabular}{|l|}
$\mathrm{T}$ \\
\end{tabular} & D & $\pi$ & $\mathrm{N}$ & $\bar{E}$ & $\mathrm{I}$ & $\mathrm{Y}$ & \begin{tabular}{|l|}
$\mathrm{E}$ \\
\end{tabular} & \begin{tabular}{l|l}
1 & 1 \\
\end{tabular} & 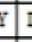 & 00 & $0 \mathrm{~N}$ & $\mathrm{D}$ & $\mathrm{U}$ & $\mathrm{N}$ & $D$ & $\mathrm{v}$ & $\mathrm{N}$ & $D$ & $\mathrm{v}$ & $\mathrm{N}$ & $\mathrm{E}$ & $\mathrm{U}$ & $\mathrm{Y}$ & 100 & 100 & 1 100 & $\mathbb{N}$ \\
\hline 27 & D & $\mathrm{U}$ & $\mathrm{N}$ & $E$ & \begin{tabular}{|l|l} 
\\
\end{tabular} & $\mathrm{Y}$ & E & I & $\mathrm{r}$ & $D$ & $\mathrm{U}$ & $N$ & $E$ & $\mathrm{I}$ & $\mathrm{Y}$ & D & $\mathrm{U} \triangle \mathrm{N}$ & $\mathrm{N}$ & $\mathrm{D}$ & $\mathbb{N}$ & D & $\mathrm{U}$ & $N$ & D & $\mathrm{v}$ & $\mathrm{N}$ & $D$ & $\mathrm{v}$ & $\mathrm{Y}$ & $E$ & $\mathrm{U}$ & $\mathrm{N}$ & No & No & No & $\mathbb{N}$ \\
\hline 28 & $D$ & $\mathrm{v}$ & $\mathrm{N}$ & $\mathrm{E}$ & 0 & $\mathrm{Y}$ & $\mathrm{E}$ & I & \begin{tabular}{|l|}
$\mathrm{Y}$ \\
\end{tabular} & D & $\mathrm{v}$ & $\mathrm{N}$ & $\mathrm{E}$ & I & $Y$ & D & $0 \mathrm{~S}$ & N & 00 & $\mathrm{~N}$ & $\bar{D}$ & $\mathrm{v}$ & $\mathrm{N}$ & $D$ & $\mathrm{v}$ & $\mathrm{N}$ & $D$ & $\mathrm{v}$ & $\mathrm{N}$ & $\mathrm{E}$ & $\mathrm{v}$ & $\mathrm{N}$ & \begin{tabular}{|l} 
No \\
\end{tabular} & 100 & No & $\mathbb{N}$ \\
\hline 29 & D & $\mathrm{U}$ & $\mathrm{N}$ & $\mathrm{E}$ & 0 & $Y$ & E & I & $\mathrm{Y}$ & D & 4 & $\mathrm{~N}$ & $\mathrm{E}$ & $\mathrm{I}$ & $\mathrm{Y}$ & D & $\mathrm{U}$ & 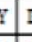 & $\mathrm{D}$ & $\mathbb{N}$ & D & $\mathrm{U}$ & $\mathrm{N}$ & D & $\mathrm{U}$ & $\mathrm{N}$ & $\mathrm{D}$ & $\mathrm{v}$ & $\mathrm{Y}$ & $\mathrm{E}$ & $\mathrm{U}$ & $\mathrm{N}$ & No & 10 & No & $\mathbb{N}$ \\
\hline 30 & $D$ & $\mathrm{U}$ & $\mathrm{N}$ & $E$ & I & $\mathrm{Y}$ & E & I & $\mathrm{Y}$ & D & $\mathrm{v}$ & $\mathrm{N}$ & $\bar{E}$ & I & $\mathrm{Y}$ & E & 0. & $\mathrm{~N}$ & 00 & $\begin{array}{l}0 N \\
N\end{array}$ & D & $\mathrm{U}$ & $\mathrm{N}$ & D & $\mathrm{v}$ & $\mathrm{N}$ & $D$ & $\mathrm{v}$ & $\mathrm{N}$ & $\mathrm{E}$ & $\mathrm{v}$ & $\mathrm{Y}$ & 10 & 100 & No & $\mathbb{N}$ \\
\hline
\end{tabular}

$P=\gamma(Y, X)=\frac{\left|P_{Y} X\right|}{|U|}$

where:

Positiveregion $\operatorname{POS}_{Y}(X)=\bigcup_{X \in U / X} \underline{Y}(Z)$

If $\mathrm{P}=1, \mathrm{X}$ totally depends on $\mathrm{Y}$, if $0<\mathrm{P}<1, \mathrm{X}$ partially depends on $\mathrm{Y}$ and if $\mathrm{P}=0, \mathrm{X}$ does not depends on $\mathrm{Y}$. So Degree of dependency $(\mathrm{P}=1)$ means the set is crisp with respect to conditional attributes (Y), otherwise set is rough. 
The block diagram of proposed classification method for detection of MI from ECG signal is shown in Figure 8 which includes of preprocessing, feature extraction and classification. The preprocessing includes filtering such as power line interference and baseline wandering by FIR and median filter (Halder et al, 2016).

The robustness of the proposed system is justified by the 'train- and- test' validation technique using Rough set Explorer system (RSES) shown in Figure 9. The knowledge is extracted from the training table and then performances (accuracy, sensitivity, and specificity) are evaluated by the testing table. The performance measures are defined as follows (Han et al, 2006; Jager et al, 1992):

Figure 8. Block diagram of MI detection method

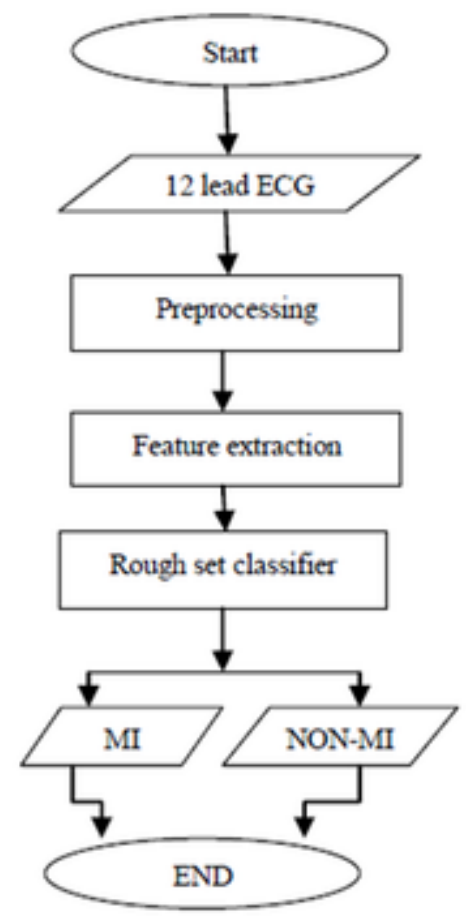

Figure 9. Confusion matrix for MI detection

\begin{tabular}{|c|c|c|c|c|c|c|}
\hline & \multicolumn{6}{|c|}{ Predicted } \\
\hline \multirow{5}{*}{ Actual } & & & & & \multirow[b]{2}{*}{ Accuracy } & \multirow[b]{2}{*}{ - } \\
\hline & & MI & NONMI & No. of obj. & & \\
\hline & MI & 135 & 3 & 138 & 0.978 & $\equiv$ \\
\hline & NONMI & 0 & 49 & 49 & 1 & $\nabla$ \\
\hline & \multicolumn{4}{|l|}{4} & \multicolumn{2}{|c|}{$D$} \\
\hline \multicolumn{7}{|c|}{ 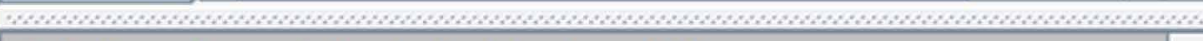 } \\
\hline \multirow{2}{*}{\multicolumn{6}{|c|}{$\begin{array}{l}\text { Total number of tested objects: } 187 \\
\text { Total accuracy: } 0.984\end{array}$}} & А \\
\hline & & & & & & $\nabla$ \\
\hline
\end{tabular}


Accuracy $=\frac{T P+T N}{T P+F N+F P+T N}$

Sensitivity $=\frac{T P}{T P+F N}$

Specificity $=\frac{T N}{T N+F P}$

where number of true positives, false positives, true negatives and false negatives for a classifier are marked by the TP, FP, TN and FN respectively.

Finally, the performance measure given by the average values of specificity, sensitivity and accuracy compares the existing methods and our experimental evaluation yielded highest classification performance of $99.27 \%$ accuracy, $99.59 \%$ sensitivity and $98.4 \%$ specificity shown in Table 5.

In the proposed system we considered the inverse problem in cardiology in term of localizing of MI region from remote voltage measurements and we think that our system may investigate the localization of MI region successfully.

\subsubsection{Classifier Quality}

This section presents the quality of the classifier generated by decision system presented in the paper. The efficiency of our classifier is reported by comparing the classification accuracy of the presented system with the different studies. For better comparison, we present these result in Table 6 .

Banerjee et al. (2014) had reported cross wavelet transform (XWT) technique based on the spectral differences and clinical features such as ST elevation, T inversion and Q wave of the ECG signals and reached $97.6 \%$ classification accuracy. Sharma et. al. (2015) used multiscale energy and eigenspace (MEES) and SVM classifier with RBF kernel for MI detection and Localization and got $96 \%$ classification accuracy. In a more recent paper (Seenivasagam et al, 2016) compared the performance of feed-forward neural network (FFNN), CCNN, and SVM with RBF kernel function for MI prediction and got $89.6 \%$ accuracy. In the same year, Hamidi et.al (2016b) applied Naive Bayes classifier and ECG features such as ST elevation and depression, and T wave inversion to detect MI events and achieved $96.5 \%$ classification accuracy. The same group applied Genetic algorithm and

Table 5. Classification result obtained from rough set decision system

\begin{tabular}{|c|c|c|c|c|c|c|c|c|c|c|c|}
\hline \multirow{2}{*}{ Classifiers } & \multicolumn{2}{|c|}{$\begin{array}{l}\text { No. of } \\
\text { Training } \\
\text { Datasets }\end{array}$} & \multicolumn{2}{|c|}{$\begin{array}{c}\text { No. of } \\
\text { Testing } \\
\text { Datasets }\end{array}$} & \multicolumn{4}{|c|}{ Detected Datasets } & \multirow{2}{*}{$\begin{array}{c}\text { Sensitivity } \\
(\%)\end{array}$} & \multirow{2}{*}{$\begin{array}{c}\text { Accuracy } \\
(\%)\end{array}$} & \multirow{2}{*}{$\begin{array}{c}\text { Specificity } \\
(\%)\end{array}$} \\
\hline & MI & $\begin{array}{c}\text { NON } \\
\text { MI }\end{array}$ & MI & $\begin{array}{l}\text { Non } \\
\text { MI }\end{array}$ & TP & $\mathbf{T N}$ & FP & FN & & & \\
\hline $\begin{array}{l}\text { Rough set } \\
\text { (Data set1) }\end{array}$ & 211 & 99 & 186 & 69 & 186 & 68 & 0 & 1 & 99.46 & 99.6 & 100 \\
\hline $\begin{array}{l}\text { Rough set } \\
\text { (Data se2) }\end{array}$ & 240 & 99 & 175 & 69 & 175 & 68 & 0 & 1 & 99.43 & 99.6 & 100 \\
\hline \multirow[t]{2}{*}{$\begin{array}{l}\text { Rough set } \\
\text { (Data set3) }\end{array}$} & 259 & 119 & 138 & 49 & 135 & 49 & 3 & 0 & 100 & 98.4 & 94.22 \\
\hline & \multicolumn{3}{|c|}{ Average result: } & & 496 & 185 & 3 & 2 & 99.59 & 99.27 & 98.4 \\
\hline
\end{tabular}


Table 6. Performance comparison for MI detection by different studies

\begin{tabular}{|c|c|c|c|c|c|c|c|c|}
\hline \multirow[b]{2}{*}{ Authors } & \multirow[b]{2}{*}{ Methods } & \multicolumn{4}{|c|}{ ECG Features } & \multicolumn{3}{|c|}{ Performances } \\
\hline & & ST Segment & T Wave & Q Wave & Others & $\begin{array}{c}\text { Accuracy } \\
{[\%]}\end{array}$ & $\begin{array}{c}\text { Sensitivity } \\
{[\%]}\end{array}$ & $\begin{array}{c}\text { Specificity } \\
{[\%]}\end{array}$ \\
\hline $\begin{array}{l}\text { Baxt et al, } \\
\text { (2002) }\end{array}$ & $\mathrm{NN}$ & $\begin{array}{l}\text { Elevation, } \\
\text { Depression }\end{array}$ & $\begin{array}{l}\text { Inversion, } \\
\text { Hyper cute }\end{array}$ & Yes & - & 98.2 & 94.5 & 95.9 \\
\hline $\begin{array}{l}\text { Arif et al, } \\
2010\end{array}$ & BPNN.PCA & deviation & amplitude & Yes & & 93.7 & 97.5 & 99.1 \\
\hline $\begin{array}{l}\text { Sun et al, } \\
(2012)\end{array}$ & LTMIL & $\begin{array}{l}\text { shape, width, } \\
\text { height }\end{array}$ & - & - & - & 90 & 91 & 85 \\
\hline $\begin{array}{l}\text { Chitra et al, } \\
\text { (2013) }\end{array}$ & $\begin{array}{l}\text { Fuzzy C } \\
\text { Means }\end{array}$ & $\begin{array}{l}\text { Elevation, } \\
\text { Depression }\end{array}$ & - & - & $\begin{array}{l}\text { Max HR } \\
\text { during } \\
\text { exercise }\end{array}$ & 92 & 91.5 & 92.1 \\
\hline $\begin{array}{l}\text { Banerjee et al, } \\
\text { (2014) }\end{array}$ & Cross WT & Elevation, & Inversion & Yes, No & & 97.6 & 97.3 & 98.8 \\
\hline $\begin{array}{l}\text { Sharma et al, } \\
\text { (2015) }\end{array}$ & MEES & Elevation & $\begin{array}{l}\text { Inversion, } \\
\text { Hyper cute }\end{array}$ & Yes & - & 96 & 93 & 99 \\
\hline $\begin{array}{l}\text { Seenivasagam } \\
\text { et al, (2016) }\end{array}$ & $\begin{array}{l}\text { CCNN, } \\
\text { FFNN, SVM }\end{array}$ & $\begin{array}{l}\text { Elevation, } \\
\text { Depression }\end{array}$ & - & - & $\begin{array}{l}\text { Max HR } \\
\text { during } \\
\text { exercise }\end{array}$ & 89.6 & 90.1 & 89.0 \\
\hline $\begin{array}{l}\text { Hamidi et al, } \\
\text { (2016a) }\end{array}$ & Naive Bayes & $\begin{array}{l}\text { Elevation, } \\
\text { Depression }\end{array}$ & Inversion & - & $\begin{array}{l}\text { Poor } \mathrm{R} \\
\text { progression }\end{array}$ & 96.5 & 95.6 & 97.7 \\
\hline $\begin{array}{l}\text { Hamidi et al, } \\
\text { (2016b) }\end{array}$ & $\begin{array}{l}\text { Genetic and } \\
\text { SVM }\end{array}$ & $\begin{array}{l}\text { Elevation, } \\
\text { Depression }\end{array}$ & Inversion & - & $\begin{array}{l}\text { Poor R } \\
\text { progression }\end{array}$ & 97.7 & 96.3 & 99.5 \\
\hline $\begin{array}{l}\text { Acharya et al, } \\
\text { (2017) }\end{array}$ & KNN & DCT coefficients & DWT coeffici & $s$ and IMFs & f EMD & 98.5 & 99.7 & 98.5 \\
\hline $\begin{array}{l}\text { Proposed } \\
\text { (2018) }\end{array}$ & $\begin{array}{l}\text { Rough set, } \\
\text { RESE }\end{array}$ & $\begin{array}{l}\text { Elevation } \\
\text { Depression, ISO }\end{array}$ & Inversion & Yes, No & $\begin{array}{l}\text { HR, QRS } \\
\text { width, QTc }\end{array}$ & 99.27 & 99.59 & 98.4 \\
\hline
\end{tabular}

SVM to the same problem in (2016a) and achieved $97.7 \%$ classification accuracy. Finally, the method in Acharya et al (2017), K-Nearest Neighbour (KNN) classifier used to achieve classification results of $98.5 \%$ accuracy and $98.5 \%$ specificity which are less of our proposed method. Hence rough set classification system has performed well in the domain of cardiovascular diseases (CVD) which yields highest classification performance of $99.27 \%$ accuracy, $99.59 \%$ sensitivity and $98.4 \%$ specificity.

\subsection{Synchronizing the Cardiologist's Phone Number With E-Mail Account Using IFTTT}

A free web-based service called IFTTT (If this then that) is used to connect the cardiologists' email accounts with their mobile phones so that every time the cardiologist gets a Dropbox notification in his mail he gets a message regarding the notification instantly. To get this service the cardiologist has to open an account on IFTTT's website shown in Figure 10.

The cardiologist then clicks on the sign-up option if he is a new user; else he clicks on the sign-in option. Then, he fills the asked details and clicks on the "Create account" option. The user then opens the website and clicks on the Sign In option. The user has to choose the type of function he wants to do. Hence, here he chooses "any new email in inbox" and "SMS" option. After clicking on the "Connect" option, we are redirected to a page where we input our phone number and get a pin. After giving our number and clicking on "Send PIN" option we get a pin which helps us to connect our mobile phones with it. After inserting the pin we click on the "Connect" option for SMS connection. Finally, we clicked on "Create Recipe" option and mail id is connected to our mobile phones and we would get a message for each Dropbox notification. 


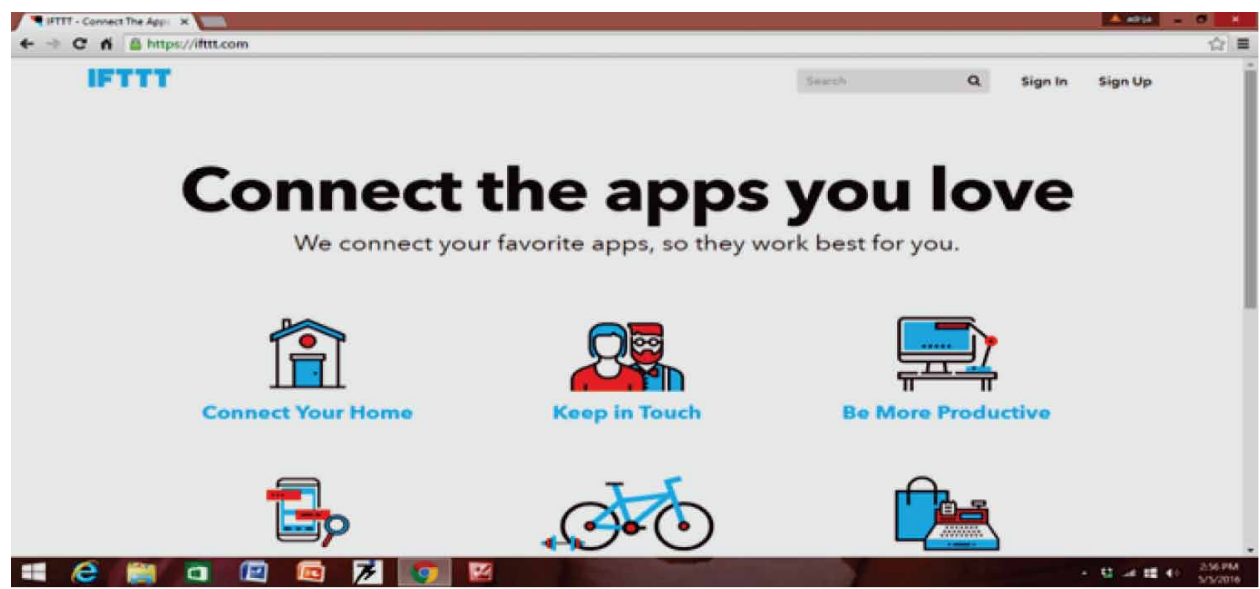

\section{CONCLUSION}

The Healthcare automation system by using cloud-based Telemonitoring technique is a rising healthcare service to offer quality patient care that may potentially transform the healthcare industry in terms of getting better entrance to patient information. In this work, we presented the rural healthcare Telemonitoring system for myocardial infarction disease classification. For MI classification, multi-dimensional data set which contains multiple objects and multiple attributes is used. Each attribute contains multiple attribute value. Due to the complexity and high dimensionality of this data set, it was a very difficult task to accurately classify of MI and Non-MI. A very good classification accuracy and sensitivity showed that proposed classification system can be trained to detect MI which will help to more accurate early diagnosis of MI. Moreover, the proposed method is simpler compared to existent methods for MI classification. A first-aid professional may be able to diagnose disease, prescribe medications, access patient's information, stored it and analyzed it to monitor and track the patients to avoid preventable death. Healthcare data are first watermarked and compressed prior to send to the cloud for safe and secure. The proposed system has been developed with the help of MATLAB, DROPBOX and IFTTT establish a worldwide connect and meets the medical needs of all people, including them who live in the most remote areas of a country. Cloud helps to use the Internet as a utility and thus all the storage and exchange of data problems are solved.

The proposed system may facilitate better care with a reduction in costs and also minimizes direct patient-cardiologist interaction. It decreases healthcare data storage place and reduces bandwidth for data transmission which brings everywhere access to quality care. Dropbox provides security to the exchange of data between the cardiologist and the health center. Because of the scalability feature of Cloud, we have to pay for only the amount of space that we use. Each patient has a separate folder with a unique id, making the managing of data easy. It has a worldwide connect. Thus, a cardiologist sitting in any corner of the world can help his patients even in remote areas, without being present there physically, with the help of his Dropbox account and internet-connected devices.

Thus, the proposed system may be preferred because it helps to save time and cost, and stores data securely in a reduced form as long as desired. It may also be helpful in making decisions in the early stage referring to the treatment of MI patients to save life and can also reduce the work load 
of cardiac specialists drastically. In near future, the proposed system may be applied for Internet of Things (IoT)-driven ECG monitoring to secure and high-quality healthcare service.

In future work, we aim to enhance the performance and consistency of our proposed work by applying deep learning technique and the detection of CAD and CHF in addition to the MI. We will also investigate the performance of this method in localizing of MI from clinical measurements. 


\section{REFERENCES}

Acharya, U. R., Fujita, H., Muhammad, A., Oh, S. L., Sudarshan, V. K., Tan, J. H., \& Tan, R. S. et al. (2017). Automated Characterization and Classification of Coronary Artery Disease and Myocardial Infarction by Decomposition of ECG Signals: A Comparative Study. Information Sciences Elsevier, 337, 17-29. doi:10.1016/j. ins.2016.10.013

Ahuja, S. P., Mani, S., \& Zambrano, J. (2012). A Survey of the State of Cloud Computing in Healthcare. Network and Communication Technologies, 1(2), 12-19.

Aranki, D., Kurillo, G., Yan, P., Bajcsy, R., \& Liebovitz, D. M. (2016), Real-Time Tele-Monitoring of Patients with Chronic Heart-Failure Using a Smartphone: Lessons Learned. IEEE Transaction on Affective Computing, 7(3).

Arif, M., Malagore, I. A., \& Afsar, F. A. (2010). Automatic detection and localization of myocardial infarction using back propagation neural networks. IEEE, 4th International Conference on Bioinformatics and Biomedical Engineering (iCBBE). doi:10.1109/ICBBE.2010.5514664

Banerjee, S., \& Mitra, M. (2014). Application of cross wavelet transform for ECG pattern analysis and classification. IEEE Transactions on Instrumentation and Measurement, 63(2), 326-333. doi:10.1109/ TIM.2013.2279001

Bashi, N., Karunanithi, M., Fatehi, F., Ding, H., \& Walters, D. (2017). Remote monitoring of patients with heart failure: An overview of systematic reviews. Journal of Medical Internet Research, 19(1), e18-e93. doi:10.2196/ jmir.6571 PMID:28108430

Baxt, W. G., Shofer, F. S., Sites, F. D., \& Hollander, J. E. (2002). A neural computational aid to the diagnosis of acute myocardial infarction. Annals of Emergency Medicine, 39(4), 366-373. doi:10.1067/mem.2002.122705 PMID:11919522

Bazan, J. G., \& Szczuka, M. (2005). The Rough Set Exploration System. In J. F. Peters \& A. Skowron (Eds.), Lecture Notes in Computer Science: Vol. 3400. Transactions on Rough Sets III. Berlin: Springer. doi:10.1007/11427834_2

Benjamin, E. J., Blaha, M. J., Chiuve, S. E., Cushman, M., Das, S. R., Deo, R., \& Muntner, P. et al. (2017). Heart Disease and Stroke Statistics-2017 Update: A Report from the American Heart Association. Circulation, 135(10), e146-e603. doi:10.1161/CIR.0000000000000485 PMID:28122885

Calabrese, B., \& Cannataro, M. (2015). Cloud computing in healthcare and biomedicine', Scalable Computing. Practice and Experience, 16(1), 1-18.

Chitra, R., \& Seenivasagam, V. (2013). Heart attack prediction system using Fuzzy C Means classifier. IOSR J. Comput. Eng., 14, 23-31.

Chow, R., Golle, P., Jakobsson, M., Shi, E., Staddon, J., Masuoka, R., \& Molina, J. (2009). Controlling data in the cloud: outsourcing computation without outsourcing control. In Proceedings of the 2009 ACM Workshop on Cloud Computing Security, CCSW '09. ACM. doi:10.1145/1655008.1655020

Dey, N., Ashour, A. S., Chakraborty, S., Banerjee, S., Gospodinova, E., Gospodinov, M., \& Hassanien, A. E. (2017). Watermarking in Biomedical Signal Processing. In Intelligent Techniques in Signal Processing for Multimedia Security. Springer International Publishing. doi:10.1007/978-3-319-44790-2_16

Ekanayake, J., Gunarathne, T., \& Qiu, J. (2011). Cloud Technologies for Bioinformatics Applications. IEEE Transactions on Parallel and Distributed Systems, 22(6), 998-1011. doi:10.1109/TPDS.2010.178

Goldberger, A.L. (2002). Clinical Electrocardiography (6 $6^{\text {th }}$ ed.). Mosby.

Halder, B., Bose, S. S., Mishra, N., \& Mitra, S. (2014). Embedding and retrieving patient's identification and compression of ECG signal. In Proceeding IEEE Conference in IIT Kharagpur 2014. West Bengal, India: IEEE.

Halder, B., \& Mitra, S. (2014). Patient Information implantation and reclamation from compressed ECG signal by LSB watermarking technique. International Journal of Emerging Technology and Advanced Engineering, 4(7), 5-15. 
Halder, B., \& Mitra, S. (2015). Modified Watermarked ECG signals by Using Adaptive Normalization Factor. In 2015 IEEE 2nd International Conference on Recent Trends in Information Systems (ReTIS). Jadavpur University.

Halder, B., Mitra, S., \& Mitra, M. (2016). Detection and Identification of ECG waves by Histogram approach. In Proceeding of the 2016 2nd International Conference on Control, Instrumentation, Energy \& Communication (CIEC16). IEEE.

Halder, B., Mitra, S., \& Mitra, M. (2018). Automatic feature extraction of ECG signal based on adaptive window dependent differential histogram approach and validation with CSE database, Int. J. Computing Systems in Engineering, 4(2/3), 146-155. doi:10.1504/IJCSYSE.2018.091394

Halder, B., Mitra, S., \& Mitra, M. (2019). Development of Cardiac Disease Classifier Using Rough Set Decision System. Emerging Technologies in Data Mining and Information Security. Advances in Intelligent Systems and Computing, 813, 775-785. doi:10.1007/978-981-13-1498-8_68

Hamidi, H., \& Daraei, A. (2016). Analysis and evaluation of techniques for myocardial infarction based on genetic algorithm and weight by SVM. J. Inf. Syst. Telecommun., 4, 85-91.

Hamidi, H., \& Daraei, A. (2016). A new hybrid method for improving the performance of myocardial infarction prediction. J. Community Health Res, 5, 110-120.

Han, J., \& Kamber, M. (2006). Data Mining: Concepts and Techniques (2nd ed.). Morgan Kaufmann.

Heart Disease Facts \& Statistics. (n.d.). Retrieved from https://www.cdc.gov/heart disease/ facts.htm

Ibaida, A., \& Khalil, I. (2013). Wavelet-Based ECG Steganography for Protecting Patient Confidential Information in Point-of-Care Systems. IEEE Transactions on Biomedical Engineering, 60(12), 3322-3330. doi:10.1109/ TBME.2013.2264539 PMID:23708767

Jager, F., Moody, G., Taddei, A., \& Mark, R. (1992). Performance measures for algorithms to detect transient ischemic ST segment changes. Computers in Cardiology, 369-372.

Liji, C. A., Indiradevi, K. P., \& Babu, K. A. (2016). Integer-to-integer wavelet transform based ECG steganography for securing patient confidential information. Procedia Technol., 24, 1039-1047. doi:10.1016/j.protcy.2016.05.230

Lin, C., Abdul, S. S., Clinciu, D. L., Scholl, J., Jin, X., Lu, H., \& Li, Y. et al. (2014). Empowering village doctors and enhancing rural healthcare using cloud computing in a rural area of mainland China. Computer Methods and Programs in Biomedicine, 113(2), 585-592. doi:10.1016/j.cmpb.2013.10.005 PMID:24296075

Lin, Y., Yu, C., \& Lin, Y. (2013). Enabling Large-Scale Biomedical Analysis in the Cloud. BioMed Research International, 2013, 1-6. PMID:24288665

Mohammed, J., Thakral, A., Ocneanu, A. F., Jones, C., Lung, C.-H., \& Adler, A. (2014). Internet of Things: Remote Patient Monitoring Using Web Services and Cloud Computing. In Proc. IEEE Internet of Things (iThings), 2014, (pp. 256-263). Taipei: IEEE.

Mouleeswaran, S. K., Rangaswamy, A., \& Abdul, R. H. (2012). Harnessing and Securing Cloud in Patient Health Monitoring. International Conference on Computer Communication and Informatics, 10-12. doi:10.1109/ ICCCI.2012.6158924

Øhrn, A. (2000). The ROSETTA C++ Library: Overview of Files and Classes', Department of Computer and Information Science, Norwegian University of Science and Technology. Trondheim, Norway: NTNU.

Pandey, S., Voorsluys, W., Niu, S., Khandoker, A., \& Buyya, R. (2012). An autonomic cloud environment for hosting ECG data analysis services. Future Generation Computer Systems, 28(1), 147-154. doi:10.1016/j. future.2011.04.022

Schamroth, L. (2009). An introduction to Electro cardiograpy (7th ed.). Blackwell Publisher.

Seenivasagam, V., \& Chitra, R. (2016). Myocardial infarction detection using intelligent algorithms. Neural Network World, 26(1), 91-110. doi:10.14311/NNW.2016.26.005

Sharma, L. N., Tripathy, R. K., \& Dandapat, S. (2015). Multiscale Energy and Eigenspace Approach to Detection and Localization of Myocardial Infarction. IEEE Transactions on Biomedical Engineering, 62(7), 1827-1837. doi:10.1109/TBME.2015.2405134 PMID:26087076 
Sun, L., Lu, Y., Yang, K., \& Li, S. (2012). ECG analysis using multiple instance learning for myocardial infarction detection. IEEE Transactions on Biomedical Engineering, 59(12), 3348-3356. doi:10.1109/TBME.2012.2213597 PMID:22929363

Tseng, K. C., \& Wu, C. C. (2014). An Expert Fitness Diagnosis System Based on Elastic Cloud Computing. The Scientific World Journal, 2014, 1-11. PMID:24723842

Wang, H., Zhang, W., \& Yu, N. (2016). Protecting patient confidential information based on ECG reversible data hiding. Multimedia Tools and Applications, 75(21), 13733-13747. doi:10.1007/s11042-015-2706-2

World Health Organization (WHO). (2017). Global health estimates: Deaths by cause, age, sex and country, 2000-2015. Retrieved from http://www.who.int/healthinfo/global_burden_disease/estimates/en/index1.html

Basudev Halder received his B.E (Computer Science \& Engg) and M.E (Computer Science \& Engg) from University of Jadavpur, West Bengal, India. At present, he is an Assistant Professor at Computer Sc \& Engg department of Neotia Institute of Technology, Management and Science, Jhinga, West Bengal (India). He has been pursuing his Ph.D. (Tech.) degree from the University of Calcutta, India. His research interests in the area of Biomedical Signal Processing. Research interest include embedding and retrieving patient's information within ECG signal, feature extraction from ECG signal, Classification and identification of heart disease from the ECG signal.

Sucharita Mitra received her B.Sc. Physics (H), M.Sc. in Electronic Science and Ph.D. (Tech) degree from University of Calcutta. At present, she is an Assistant Professor at the Electronics Department of Netaji Nagar Day College. Formerly, she was attached to the Indian Statistical Institute as a Post Doctoral Fellow and the Department of Applied Physics (CU) as a Guest Faculty. She visited University of Oxford as a PDF under the DBT-CREST award scheme of GOI. Her research interests include biomedical signal and image processing, telemedicine and mobile ECG, pattern recognition, etc. She has published more than 55 research papers in reputed international and national journals and conference proceeding.

Madhuchhanda Mitra received the B.Sc. degree in Physics (with honors), and the B. Tech, M.Tech, and Ph.D. degree in measurement and instrumentation from the University of Calcutta, Calcutta, India. She has 23 years research and 18 years teaching experience in the University college of Technology, University of Calcutta. Her special fields of interest include biomedical signal processing, process instruments, microprocessors, and microcontroller. She is currently a Professor with the Instrumentation Engineering Division, Department of Applied Physics. She has about 145 technical papers published in national and international journals and conference proceedings. Dr. Mitra is a recipient of "Griffith Memorial Award" of the University of Calcutta. 